PSICOLOGÍA

IBEROAMERICANA
Psicología Iberoamericana ISSN: 1405-0943

revista.psicologia@ibero.mx

Universidad Iberoamericana, Ciudad de México México

\title{
Inteligencia emocional en los psicólogos en formación
}

Castillo Castañeda, Georgina; Pérez-Sánchez, Lucía; Becerra-Altamirano, Nydia Kisaí

Inteligencia emocional en los psicólogos en formación

Psicología Iberoamericana, vol. 29, núm. 2, 292403, 2021

Universidad lberoamericana, Ciudad de México, México

Disponible en: https://www.redalyc.org/articulo.oa?id=133967822007

DOI: https://doi.org/10.48102/pi.v29i2.403

Los autores/as conservan los derechos de autor y ceden a la revista el derecho de la primera publicación, con la obra registrada bajo la Licencia Creative Commons Atribución 4.0 Internacional.

Los autores/as conservan los derechos de autor y ceden a la revista el derecho de la primera publicación, con la obra registrada bajo la Licencia Creative Commons Atribución 4.0 Internacional,

\section{(c) (1)}

Esta obra está bajo una Licencia Creative Commons Atribución 4.0 Internacional. 


\title{
Inteligencia emocional en los psicólogos en formación
}

\author{
Emotional intelligence in trainee psychologists \\ Georgina Castillo Castañeda gina.castillo@uan.edu.mx \\ Universidad Autónoma de Nayarit, México \\ (D) https://orcid.org/0000-0001-6358-550X \\ Lucía Pérez-Sánchez lucia.perez@uan.edu.mx \\ Universidad Autónoma de Nayarit, México \\ (D) https://orcid.org/0000-0003-1614-7587 \\ Nydia Kisaí Becerra-Altamirano nydia.becerra@uan.edu.mx \\ Universidad Autónoma de Nayarit, México \\ https://orcid.org/0000-0003-2452-5365
}

Psicología Iberoamericana, vol. 29, núm. 2, 292403, 2021

Universidad Iberoamericana, Ciudad de México, México

Recepción: 14 Junio 2021

Aprobación: 02 Noviembre 2021

DOI: https://doi.org/10.48102/ pi.v29i2.403

Redalyc: https://www.redalyc.org/ articulo.oa?id=133967822007
Resumen: La presente investigación tuvo por objetivo comparar la inteligencia emocional de 239 estudiantes del primer y último año de la carrera de Psicología de la Universidad Autónoma de Nayarit, estableciendo diferencias por sexo y grado con el afán de profundizar en los procesos de formación del psicólogo en la universidad pública. Se utilizó un muestreo no probabilístico por conveniencia, manteniendo un enfoque cuantitativo, de corte transversal, descriptivo y comparativo. Se aplicó el Inventario de Manejo Emocional, obteniendo una consistencia interna satisfactoria en sus tres dimensiones (.83 - .91), utilizando la prueba $U$ de Mann Whitney para comparar el manejo emocional, tanto entre los grupos de primer y último año, como entre hombres y mujeres. Los hallazgos demuestran que son las emociones de empatía y equilibrio emocional aquellas que en mayor medida se ven influenciadas por el grado escolar que cursan los participantes, concluyendo que existe un decremento en las valoraciones de los factores Afrontamiento emocional y Emociones positivas en los estudiantes de Psicología a medida que se avanza en los estudios profesionales.

Palabras clave: universitarios, enseñanza de la psicología, emociones, perfil emocional, empatía.

Abstract: The objective of this research was to compare the emotional intelligence of 239 students in the first and last year of the Psychology career at the Autonomous University of Nayarit, establishing differences by sex and grade to deepen the training processes of the psychologist in the public university. Non-probabilistic convenience sampling was used, maintaining a quantitative, cross-sectional, descriptive and comparative approach. The Emotional Management Inventory was applied, obtaining a satisfactory internal consistency in its three dimensions $(.83-.91)$, using the Mann Whitney $U$ test to compare emotional management, both between the first and last year groups, and between men and women. The findings show that the emotions of empathy and emotional balance are those that are influenced to a greater extent by the school grade that the participants study. In conclusion, there is a decrease in the evaluations of the factors "emotional coping and positive emotions" in psychology students as you advance in professional studies.

Keywords: college students, educational psychology, emotions, emotional profile, empathy. 


\section{Introducción}

En el plano escolar, desde el nivel básico al universitario, tradicionalmente se ha orientado la formación académica hacia la búsqueda de las mejores notas escolares y la acumulación de conocimiento como referente de inteligencia, traducida como aspecto esencial para garantizar el éxito académico, profesional y personal (Delors et al., 1997; Fragoso-Luzuriaga, 2015; Secretaría de Educación Pública, 2017; Vivas García, 2003). Ello ha provocado se dejen de lado habilidades de carácter social y emocional que permiten el correcto desarrollo del individuo en el centro escolar y su participación en la vida social. En este sentido, algo que ha motivado la búsqueda del impacto de la inteligencia emocional en el rendimiento académico y en variables como la motivación y las actitudes ha sido la identificación de competencias emocionales como elementos determinantes para la adaptación de las personas a la sociedad (Del Rosal Sánchez et al., 2016; Paez Cala \& Castaño Castrillón, 2015).

En este sentido, ha quedado claro que la inteligencia emocional no debe ser excluida de los procesos de formación académica formal (Cardona Carmona, 2008) y debe trabajarse tanto dentro como fuera de los escenarios escolares (Gutiérrez Carmona \& Expósito López, 2015) desde la perspectiva cognitiva y personal (Castillo Castañeda et al., 2016). Conceptualmente, la inteligencia emocional se ha entendido como la habilidad que desarrollan las personas para percibir los sentimientos propios y de los demás, la capacidad de comprenderlos y regularlos, así como modificarlos en función del estado de ánimo personal o el de los demás (Cazalla-Luna \& Molero, 2016). De manera agregada, hay quienes la visualizan como un rasgo a desplegar en la persona, identifican aspectos asociados a la atención, la claridad de experiencias y la regulación como aspectos esenciales del desarrollo emocional (Antonio-Agirre et al., 2015; Calero et al., 2018; Carpio Fernández et al., 2019).

Lo anterior, indica que la inteligencia emocional habrá de desarrollarse a través de ejercicios de introspección y extrospección del sujeto hacia el reconocimiento de sus fortalezas y áreas de oportunidad en diversos ámbitos de su vida (académica, social, emocional, familiar y física, por mencionar algunas), dicho proceso Salovey y Mayer (1990, p. 189) definen como "la capacidad para supervisar los sentimientos y las emociones de uno/a mismo/a y de los demás, de discriminar entre ellos y de usar esta información para la orientación de la acción y el pensamiento propios". Por lo que en este estudio empleamos el término de manejo emocional como uno de los elementos de la inteligencia emocional, propuestos en 1990 por Salovey y Mayer. Por su parte, Gutiérrez Carmona y Expósito López (2015, p. 44) la visualizan como aquella que permite "tomar conciencia de nuestras emociones, comprender los sentimientos de los demás, tolerar las presiones y frustraciones que soportamos en el trabajo, acentuar nuestra capacidad de trabajar en equipo y adoptar una actitud empática y social". 
En el caso del escenario escolar, Fernández-Berrocal y Extremera (2002, p. 2) reconocen la inteligencia emocional como una habilidad esencial a desarrollar en la escuela. A saber, como la:

...habilidad de las personas para atender y percibir los sentimientos de forma apropiada y precisa, la capacidad para asimilarlos y comprenderlos de manera adecuada y la destreza para regular y modificar nuestro estado de ánimo o el de los demás (p. 2).

Reconociendo cuatro componentes en su configuración: la percepción y expresión emocional, facilitación emocional, comprensión emocional y regulación emocional (Fernández-Berrocal \& Extremera, 2002).

Con base en la revisión documental de estudios asociados a la inteligencia emocional, la evidencia empírica señala que los hombres presentan mejores valoraciones al momento de percibir, regular y reparar los estados de ánimo negativo (Calero et al., 2018; Del Rosal Sánchez et al., 2016; Pérez-Bonet \& Velado-Guillén, 2017) mientras que las mujeres prestan mayor atención, tienen mejor claridad y pueden expresar de mejor manera sus estados emocionales (Gartzia et al., 2012; Suárez Colorado et al., 2012; Suárez Colorado \& Wilches Bisval, 2015; Valadez Sierra et al., 2013).

En este sentido, otros autores, como Santoya Montes et al. (2018) han optado por estudiar lo que denominan autoconocimiento emocional como un factor asociado a la capacidad de autorregulación emocional, entendido como "la capacidad de ser consciente de las propias emociones, conocerlas y entenderlas" (p. 424). De forma similar Castillo Castañeda et al. (2016) y Rábago de Ávila et al. (2019) trabajan el concepto de inteligencia emocional, como manejo emocional en el que se destaca el género como un factor importante para el afrontamiento emocional y la presencia de niveles de riesgo en cuanto a la presencia de las emociones positivas: alegría, tranquilidad, satisfacción y seguridad en estudiantes de psicología. Castillo Castañeda et al. (2016, p. 141) conceptualizan el manejo emocional como "la habilidad que poseen las personas para emplear sus recursos en la solución de problemas, identificar sus emociones (sean éstas positivas o negativas) y comunicarlas de forma eficiente".

Lo anterior, indica que las habilidades socioemocionales son indispensables para cualquier persona en distintas áreas de su vida. En el caso específico del profesional de la Psicología, las dimensiones personales juegan un papel fundamental en el saber hacer, ya que contribuyen al éxito de las intervenciones psicológicas. Por ejemplo, se ha encontrado que en el área de la clínica se han realizado mayores exploraciones y producciones sobre las dimensiones personales implicadas en el rol del psicólogo (González-Serna et al., 2014), mientras que las investigaciones referidas a la efectividad en la psicoterapia, señalan que los factores comunes o inespecíficos de la relación terapéutica, resultan más relevantes que los resultados de la teoría y técnica que los terapeutas utilizan (Best \& Pairetti, 2014a).

De ahí la importancia de que los psicólogos en formación desarrollen las habilidades asociadas a la inteligencia emocional, pues 
así también, en la experiencia de los tutores-profesores del programa de psicología, manifiestan la necesidad constante de sus estudiantes, de recibir orientación psicopedagógica, argumentando que muchas de sus dificultades radican en el deficiente manejo de sus habilidades emocionales, los cuales impactan las áreas personal y académica. Surge la pregunta, ¿hay diferencias significativas de inteligencia emocional entre los estudiantes de primero y cuarto año?; ¿hay diferencias significativas de inteligencia emocional por sexo entre los estudiantes de primero y cuarto año?, por lo que surgen las hipótesis:

$H i$. Existen diferencias significativas de inteligencia emocional entre los estudiantes de primer y cuarto año de la carrera de psicología, así como tambien diferencias significativas por sexo.

Ho. No existen diferencias significativas de inteligencia emocional entre los estudiantes de primer y cuarto año de la carrera de psicología, así como tampoco existen diferencias significativas por sexo.

En la literatura especializada existen numerosos trabajos asociados a la formación profesional del psicólogo y el desarrollo de competencias específicas para desempeñarse en contextos formales y no formales. A saber, en campos específicos de la atención clínica y de salud (Belloch Fuster, 2005; Echeburúa et al., 2005, 2012), de la atención familiar (Gutiérrez Rodríguez et al., 2018; Rodrigo López, 2017), del desarrollo comunitario (Serrano-García, 2015), de la educación (Barraza-López, 2015; Jiménez González \& Báez, 2017; Pérez-González \& García-Ros, 2017), de la investigación (Bello-Carrasco \& Villavicencio-Navia, 2015) y del campo laboral (Ruiz et al., 2008) por mencionar los principales escenarios profesionales en los que se busca insertar y para los que se establecen diversas competencias a desarrollar durante la formación académica. Best y Pairetti (2014b) han trabajado la importancia del desarrollo de la inteligencia emocional durante el proceso de formación profesional del psicólogo, así también las investigaciones de Castillo Castañeda (2016), Castillo Castañeda et al. (2016) y Rábago de Ávila et al. (2019).

Por otra parte, Cabrera et al., (2010) han documentado las competencias que se deben desarrollar el Psicólogo en su fase formativa en la universidad. Ligado al tema de inteligencia emocional, los autores han identificado dentro de las competencias instrumentales la toma de decisiones y resolución de problemas, para el caso de las competencias interpersonales destacan el trabajo en equipo, las habilidades sociales y el reconocimiento de la diversidad como las más relevantes de esta competencia, mientras que para el caso de la competencia sistémica la capacidad de adaptación a los cambios es la que repercute directamente hacia la inteligencia emocional.

Con relación al rol del profesional de la Psicología, se destacan aspectos tales como el autoconocimiento, la empatía, la capacidad de escucha, el manejo paciente-psicólogo y la reflexión sobre el rol profesional. A saber, el estudiantado universitario de la carrera de Psicología al igual que los profesionales graduados que se encuentran ejerciendo la profesión entorno al manejo de las emociones, destacan la capacidad de escuchar, 
mientras que el profesorado, considera de suma importancia la relación paciente-psicólogo y la capacidad de adaptación a situaciones nuevas (Bohart \& Greenberg, 1997 y Norcross \& Wampold, 2011 en Muñoz Aguirre, 2020).

Ante lo anterior, Pérez-González y García-Ros (2017) añaden como un gran problema de formación la gran cantidad de funciones y actuaciones que se le asignan al profesional de la Psicología (por ejemplo, dificultades de aprendizaje, altas capacidades, trastornos del desarrollo, problemas conductuales, de rendimiento y abandono académico temprano, prevención del suicidio, trastornos y problemas emocionales, entre otros), cuestión que lo puede conducir a tener problemas de formación durante el programa universitario.

Respecto a lo anterior, Barón y Benítez (2020, p. 124) al analizar las exigencias de la profesión del psicólogo en el ámbito emocional expresa que el desarrollo de esta competencia supone una serie de consecuencias, ya que requiere "plantear un conjunto de saberes (saber, saber hacer, saber estar y saber ser-conocimientos, procedimientos y actitudes) combinados, coordinados e integrados en el ejercicio profesional". Por otra parte, Serrano-García (2015) en la cuestión valoral del psicólogo profundiza en lo que considera valores fundamentales del ejercicio profesional, entre los que destacan: la promoción de la justicia social (entendida como la búsqueda del bienestar), la investigación participativa, la diversidad cultural (entendida como el respeto y la aceptación de todos los individuos) y la prevención primaria de la salud. Por lo tanto, además de tener una formación académica especializada, se requiere que el estudiantado de Psicología desarrolle habilidades sociales y emocionales que le permitan trabajar en equipo, aprender a mirar y escuchar, así como empatizar, comprender y analizar (Ceberio \& Linares, 2005; Echeburúa et al., 2005; Fabregó Claparols \& Viñas Millán, 2010).

De la misma manera, pero en el plano escolar, Barraza-López (2015) hace mención de cuatro pilares relacionados a las competencias emocionales del psicólogo: 1. Las habilidades sociales, emocionales e intelectuales, 2. La convivencia social y salud mental, 3. La inclusión y la diversidad, y 4. La gestión curricular de aula y escolar de calidad. En este sentido, cuando se refiere al psicólogo expresa que debe desarrollar acciones específicas a estas áreas. A saber, para el caso de las habilidades sociales, emocionales e intelectuales sugiere promover la reflexión conjunta de los actores de la unidad educativa en torno a cuestiones como el respeto, la empatía, la colaboración y la solidaridad. En cuanto a convivencia social y salud mental, debe promover el bienestar personal y colectivo, tanto dentro como fuera del currículo educativo. En lo referido a inclusión y la diversidad, debe desarrollar espacios de tolerancia, la valoración de la diversidad y el desarrollo de los talentos. Por último, con relación a la gestión de aula y escolar de calidad, promover la orientación, el acompañamiento y los procesos de adaptación curricular.

Puede decirse entonces que educar emocionalmente es hacer consciente el hecho de que el ser humano no sólo tiene necesidades físicas por saciar, sino que también debe satisfacer cuestiones emocionales 
para responder ante el presente inmediato y para proyectarse hacia un futuro en su proceso de autorrealización. En este sentido, Castillo Castañeda (2016) expresa que dichas necesidades el ser humano las resuelve expresando dos tipos de emociones: positiva y negativa. A saber, Alegría, Tranquilidad, Satisfacción y Seguridad en el polo positivo, mientras que de manera negativa se expresa Tristeza, Enojo, Apatía, Inseguridad y Ansiedad. En este sentido, se trabaja en función de los que Fragoso-Luzuriaga (2015) reconoce como una competencia emocional, entendida como el conjunto de capacidades y habilidades que permite a las personas desarrollarse en ambientes cambiantes, como aquellas que permiten adaptarse y a la vez, visualizarse como una persona diferente, que en términos positivos, presente mayor confianza en sí misma.

Los referentes anteriores, asociados a la inteligencia y el desarrollo de habilidades socioemocionales, dan cuenta de la necesidad de formar a los estudiantes de todos los niveles educativos en inteligencia emocional.

\section{Método}

\section{Participantes}

Se obtuvo una muestra no probabilística de tipo intencional de 239 estudiantes voluntarios de la Licenciatura en Psicología de una Institución de Educación Pública del estado de Nayarit, México. Particularmente, el $74.1 \%$ eran mujeres y $25.9 \%$ hombres, con edades entre los 18 y 53 años $(M=20.5, D E=4.3)$. Con respecto al grado en curso, el 68.2\% (163) eran estudiantes de recién ingreso, es decir, que se encontraban dentro del primer año de la carrera, mientras que $31.8 \%$ (76) se encontraban en último año, a saber, a punto de egresar de la licenciatura.

\section{Instrumentos}

\section{Inventario de manejo emocional}

Inventario de manejo emocional. Diseñado y validado por Castillo Castañeda (2016) y posteriormente adaptado por Castillo Castañeda et al. (2016) en universitarios durante su trayecto escolar. Se encuentra constituido por 242 reactivos y tres dimensiones: Recursos para la solución de problemas, Emociones positivas y Emociones negativas. A su vez, cada dimensión se compone por una serie de escalas. La Tabla 1 muestra su constitución específica. Los reactivos tienen un formato de respuesta dicotómico (Sí- No). Para obtener el puntaje para cada escala, se suman la cantidad de reactivos que fueron contestados afirmativamente, se divide entre el total de ítems y se multiplica por 100, obteniendo porcentajes para cada escala (el puntaje final puede oscilar entre 0 y 100). Para las escalas que componen las dimensiones de Recursos para la Solución de Problemas y Emociones positivas, un promedio elevado será sinónimo 
de un mejor manejo emocional, por el contrario, para las que componen el factor de Emociones negativas, un puntaje mayor implica un peor manejo emocional. Como medida adicional, se estableció en cada una de las escalas una clasificación ordinal de cuatro categorías a partir de los percentiles, considerando el grado de riesgo implicado en cada nivel (Riesgo, Medio bajo, Medio alto y Deseable). Diversos estudios han confirmado propiedades psicométricas satisfactorias para el Inventario de manejo emocional (Castillo Castañeda, 2016), específicamente, en este estudio se encontraron valores satisfactorios de consistencia interna (KR-20) en la dimensión de Recursos para la solución de problemas (.84), Emociones positivas (.83) y Emociones negativas (.91). 


\section{Tabla 1}

Tabla 1

Dimensiones y escalas del Inventario de Manejo Emocional

\begin{tabular}{ccl}
\hline Dimensiones y escalas & Cantidad de ítems & Ejemplo de reactivo \\
\hline $\begin{array}{l}\text { Recursos para la solución de } \\
\text { problemas }\end{array}$ & 20 & $\begin{array}{l}\text { Algunas personas se acercan a mí porque perciben que puedo entenderlas y } \\
\text { apoyarlas en sus dificultades. }\end{array}$ \\
\hline Empatía & 20 & $\begin{array}{l}\text { Me describo como una persona que siempre está atenta a lo que sucede a su } \\
\text { alrededor. }\end{array}$ \\
Equilibrio emocional & 20 & $\begin{array}{l}\text { Con facilidad me recupero emocionalmente después de un duro golpe o ante } \\
\text { la adversidad. }\end{array}$ \\
Radurez emocional & 18 & $\begin{array}{l}\text { Me permito sentir mis emociones, sin tratar de evitarlas y sin actuar en } \\
\text { consecuencia a ellas. }\end{array}$
\end{tabular}

Emociones positivas

\begin{tabular}{|c|c|c|}
\hline Alegría & 16 & Tengo un buen estado de ánimo la mayor parte del tiempo. \\
\hline Tranquilidad & 19 & Ante una situación molesta procuro mantener la calma. \\
\hline Satisfacción & 20 & Siento que he disfrutado cada etapa que he vivido. \\
\hline Seguridad & 19 & Me siento seguro(a) cuando tengo la protección de un grupo de amigos. \\
\hline \multicolumn{3}{|l|}{ Emociones negativas } \\
\hline Tristeza & 17 & Regularmente me siento con falta de ánimo o de alegría. \\
\hline Enojo & 18 & Frecuentemente hay cosas que me molestan sin tener un motivo aparente. \\
\hline Apatía & 17 & He dejado de entusiasmarme por las cosas que comúnmente me gustaban. \\
\hline Inseguridad & 20 & Me siento confundido(a) al tener que decidir entre varias opciones. \\
\hline Ansiedad & 18 & Se me dificulta dejar de pensar en las cosas que me causan preocupación. \\
\hline
\end{tabular}

Fuente. Elaboración propia con base en Castillo Castañeda, G., Arias Guzmán, A. A., \&

Ramírez Vázquez, M. E. (2016). Medición del manejo emocional en estudiantes de nivel superior y su importancia en la trayectoria escolar. Revista Educateconciencia, 12(13), 137-150. 


\section{Procedimiento y consideraciones éticas}

Se contactó a las autoridades del programa de psicología para explicarles el objetivo del estudio obteniendo la autorización para llevar adelante la investigación, posteriormente fueron contactados los tutores de cada grupo de primer y cuarto año para que cedieran a los investigadores una hora de su tiempo con el grupo. Siguiendo los principios éticos vigentes, se solicitó a los estudiantes la firma del consentimiento informado como condición excluyente para participar del estudio. Además, se les informó acerca de los objetivos de este trabajo, la confidencialidad de los datos recolectados aclarándoles que su participación era voluntaria, pudiendo abandonar la investigación en el momento que lo desearan.

A continuación, en el aula y de forma grupal los estudiantes respondieron al inventario de modo autoadministrado, durante su horario de tutoría. La administración fue supervisada por psicólogos capacitados en el uso del instrumento.

\section{Análisis de datos}

En primer lugar se determinó el tipo de herramientas estadísticas a utilizar a partir del nivel de medición del Inventario de manejo emocional. Si bien el formato de respuesta es dicotómico (nominal), al sumar los reactivos y obtener porcentajes por cada escala se obtenía un valor numérico del que podrían obtenerse promedios y desviaciones estándar. En dicho sentido, se calcularon las medias, desviaciones y frecuencias de acuerdo a los puntos de corte para cada una de las escalas. Posteriormente, se evaluó la distribución de todas las mediciones para determinar el uso de pruebas paramétricas o no paramétricas. Si bien el nivel de medición no corresponde a una escala de razón o intervalo, de encontrarse una distribución cercana a la normal podría justificarse el uso de pruebas paramétricas. Sin embargo, la prueba de Kolmogorov confirmó la ausencia de dicho supuesto $(p<.05)$, por lo que se utilizó la U de Mann Whitney para comparar el manejo emocional, tanto entre los grupos de primer y último año, como entre hombres y mujeres. En ambos casos se utilizó la $\mathrm{r}$ de Rosenthal en la estimación del tamaño del efecto.

\section{Resultados}

Para dar orden a la presentación de resultados, se exhiben en primera instancia las medias y desviaciones estándar de los distintos factores que conforman el inventario de manejo de emociones. Además, dado que los puntajes de los participantes fueron clasificados, se muestran las frecuencias y porcentajes para cada categoría (Tabla 2). 
Tabla 2

\section{Tabla 2}

Resultados descriptivos

\begin{tabular}{llllll}
\hline Nivel & \multicolumn{5}{c}{ Clasificación } \\
\hline$M$ & $D E$ & Riesgo & Medio bajo & Medio alto & Deseable
\end{tabular}

\begin{tabular}{lcccccc}
\hline Afrontamiento emocional & \multicolumn{1}{l}{} \\
\hline Empatía & 74.27 & 17.40 & $7(2.9 \%)$ & $54(22.6 \%)$ & $87(36.4 \%)$ & $91(38.1 \%)$ \\
Equilibrio Emocional & 74.90 & 18.50 & $19(7.9 \%)$ & $46(19.2 \%)$ & $106(44.4 \%)$ & $68(28.5 \%)$ \\
Resiliencia & 81.38 & 16.59 & $7(2.9 \%)$ & $64(26.8 \%)$ & $100(41.8 \%)$ & $68(28.5 \%)$ \\
Madurez emocional & 74.45 & 17.25 & $12(5.0 \%)$ & $68(28.5 \%)$ & $93(38.9 \%)$ & $66(27.6 \%)$
\end{tabular}

Emociones positivas

\begin{tabular}{lcccccc}
\hline Alegría & 73.26 & 22.98 & $14(5.9 \%)$ & $76(31.8 \%)$ & $80(33.5 \%)$ & $69(82.9 \%)$ \\
Tranquilidad & 66.90 & 18.57 & $14(5.9 \%)$ & $62(25.9 \%)$ & $78(32.6 \%)$ & $85(35.6 \%)$ \\
Satisfacción & 71.36 & 22.08 & $15(6.3 \%)$ & $76(31.8 \%)$ & $86(36.0 \%)$ & $62(25.9 \%)$ \\
Seguridad & 77.85 & 16.89 & $7(2.9 \%)$ & $64(26.8 \%)$ & $84(35.1 \%)$ & $84(35.1 \%)$
\end{tabular}

Emociones negativas

\begin{tabular}{lcccccc}
\hline Tristeza & 41.48 & 24.04 & $86(36.0 \%)$ & $71(29.7 \%)$ & $66(27.6 \%)$ & $16(6.7 \%)$ \\
Enojo & 30.46 & 20.50 & $62(25.9 \%)$ & $91(38.1 \%)$ & $58(24.3 \%)$ & $28(11.7 \%)$ \\
Apatía & 33.26 & 23.04 & $72(30.1 \%)$ & $79(33.1 \%)$ & $71(29.7 \%)$ & $17(7.1 \%)$ \\
Inseguridad & 46.95 & 24.20 & $101(42.3 \%)$ & $62(25.9 \%)$ & $65(27.2 \%)$ & $11(4.6 \%)$ \\
Ansiedad & 40.74 & 25.31 & $99(41.4 \%)$ & $59(24.7 \%)$ & $61(25.5 \%)$ & $20(8.4 \%)$ \\
\hline
\end{tabular}

Nota. En el caso de afrontamiento emocional y emociones positivas, un mayor puntaje indica un mejor manejo emocional. En el caso de emociones negativas la puntuación está invertida, un mayor puntaje indica un manejo de emociones más deficiente. Fuente. Elaboración propia.

Fuente. Elaboración propia

Nota. En el caso de afrontamiento emocional y emociones positivas, un mayor puntaje indica un mejor manejo emocional. En el caso de emociones negativas la puntuación está invertida, un mayor puntaje indica un manejo de emociones más deficiente.

Es posible apreciar que, dentro del factor de Afrontamiento emocional, Resiliencia ( $M=81.38, D E=16.59)$ obtuvo una puntuación ligeramente superior al resto, mientras que Equilibrio emocional tuvo el mayor 
porcentaje de estudiantes en situación de riesgo (7.9\%). En el caso de las Emociones positivas, Seguridad $(M=77.85, D E=16.89)$ se destacó al tener la puntuación más alta y la menor proporción de estudiantes en situación de riesgo (2.9\%). Por último, dentro de las Emociones negativas, Inseguridad ( $M=46.95, D E=24.20)$ sobresale como elemento con el manejo emocional más deficiente, así como con la mayor cantidad de estudiantes en condición de riesgo (42.3\%).

Enseguida, se compararon las diferentes dimensiones del manejo emocional entre los estudiantes de primer año o reciente ingreso y los estudiantes a punto de egresar o de último año. Debido a la ausencia de normalidad (se menciona en el apartado de análisis de datos), se utilizó para este contraste un conjunto de pruebas de $U$ de Mann Whitney junto a la $r$ de Rosenthal para la estimación del tamaño del efecto. La Tabla 3 muestra los hallazgos. 
Tabla 3

Tabla 3

Comparación por año del estudiantado

\begin{tabular}{llll}
\multicolumn{2}{l}{ Primer año } & \multicolumn{2}{l}{ Úlimo año } \\
\hline$M$ & $D E$ & $M$ & $D E$
\end{tabular}

Afrontamiento emocional

\begin{tabular}{lccccccc}
\hline Empatía & 76.56 & 16.56 & 69.34 & 18.23 & -2.96 & .003 & .19 \\
Equilibrio Emocional & 76.84 & 18.09 & 70.72 & 18.81 & -2.52 & .012 & .16 \\
Resiliencia & 82.48 & 15.99 & 79.01 & 17.68 & -1.41 & .158 & .09 \\
Madurez emocional & 74.77 & 17.73 & 73.78 & 16.26 & -0.67 & .498 & .04
\end{tabular}

Emociones positivas

\begin{tabular}{lccccccc}
\hline Alegría & 74.95 & 22.42 & 69.63 & 23.88 & -1.66 & .095 & .11 \\
Tranquilidad & 68.19 & 17.87 & 64.12 & 19.83 & -1.69 & .091 & .11 \\
Satisfacción & 72.82 & 21.76 & 68.22 & 22.59 & -1.52 & .127 & .10 \\
Seguridad & 78.39 & 16.86 & 76.70 & 17.01 & -0.88 & .376 & .06
\end{tabular}

Emociones negativas

\begin{tabular}{lcccccccc}
\hline Tristeza & 41.90 & 23.62 & 40.58 & 25.04 & -0.54 & .587 & .04 \\
Enojo & 29.48 & 20.13 & 32.58 & 21.28 & -1.13 & .258 & .07 \\
Apatía & 33.06 & 22.11 & 33.68 & 25.05 & -0.18 & .854 & .01 \\
Inseguridad & 46.56 & 24.18 & 47.76 & 24.39 & -0.40 & .684 & .03 \\
Ansiedad & 40.98 & 24.93 & 40.24 & 26.27 & -0.29 & .769 & .02 \\
\hline Fuente. & & & & & & & & \\
\hline
\end{tabular}

Fuente. Elaboración propia

Únicamente en las escalas de Empatia ( $Z=-2.96, p=.003, r=.19)$ y Equilibrio emocional $(Z=-2.52, p=.012, r=.16)$ se presentaron diferencias estadísticamente significativas, siendo los estudiantes de primer año quienes mostraron un mejor manejo, con un tamaño del efecto pequeño. Aunque solo las diferencias en tales escalas fueron significativas, llama poderosamente la atención que, en las dimensiones de afrontamiento emocional y emociones positivas, los puntajes sean mayores para los 
estudiantes de reciente ingreso en todos los casos, mientras que, en el factor de emociones negativas, solo mostraron medias ligeramente superiores en Tristeza y Ansiedad.

Posteriormente, fue ejecutada una comparación sobre los factores que conforman el manejo emocional entre hombres y mujeres. Nuevamente, dada la violación del criterio de normalidad, se utilizó un conjunto de pruebas $U$ de Mann Whitney junto a la $r$ de Rosenthal para la estimación de la magnitud del efecto. Estos hallazgos se muestran en la Tabla 4. 
Tabla 4

Tabla 4

Comparación por sexo del estudiantado

\begin{tabular}{|c|c|c|c|c|c|c|c|}
\hline & \multicolumn{2}{|c|}{ Hombres } & \multicolumn{2}{|c|}{ Mujeres } & \multirow{2}{*}{$Z$} & \multirow{2}{*}{$p$} & \multirow{2}{*}{$r$} \\
\hline & $M$ & $D E$ & $M$ & $D E$ & & & \\
\hline \multicolumn{8}{|c|}{ Afrontamiento emocional } \\
\hline Empatía & 71.07 & 19.73 & 75.45 & 16.44 & -1.37 & .169 & .09 \\
\hline Equilibrio Emocional & 79.67 & 15.96 & 73.39 & 19.03 & -2.24 & .025 & .15 \\
\hline Resiliencia & 84.59 & 15.87 & 80.25 & 16.77 & -2.18 & .029 & .14 \\
\hline Madurez emocional & 76.43 & 15.24 & 73.88 & 17.87 & -0.75 & .448 & .05 \\
\hline
\end{tabular}

Emociones positivas

\begin{tabular}{lccccccc}
\hline Alegría & 77.16 & 24.24 & 72.01 & 22.47 & -1.93 & .053 & .13 \\
Tranquilidad & 71.44 & 16.74 & 65.41 & 18.97 & -2.25 & .024 & .15 \\
Satisfacción & 74.18 & 23.27 & 70.40 & 21.70 & -1.45 & .144 & .09 \\
Seguridad & 81.64 & 16.14 & 76.54 & 17.03 & -2.34 & .019 & 15
\end{tabular}

Emociones negativas

\begin{tabular}{lcccccccc}
\hline Tristeza & 31.84 & 18.97 & 44.63 & 24.72 & -3.49 & .000 & .23 \\
Enojo & 24.84 & 18.73 & 32.42 & 20.83 & -2.57 & .010 & .17 \\
Apatía & 29.69 & 20.39 & 34.21 & 23.59 & -1.15 & .250 & .07 \\
Inseguridad & 39.34 & 22.97 & 49.46 & 24.16 & -2.85 & .004 & .18 \\
Ansiedad & 33.08 & 23.20 & 43.33 & 25.59 & -2.75 & .006 & .18 \\
\hline
\end{tabular}

Fuente. Elaboración propia

\section{Fuente. Elaboración propia}

En el factor de Afrontamiento emocional, se presentaron diferencias estadísticamente significativas en equilibrio emocional $(Z=-2.24, p=.025$, $r=.15)$ y Resiliencia $(Z=-2.18, p=.029, r=.14)$, siendo los hombres quienes presentaron niveles más adecuados. Del mismo modo, dentro de las emociones positivas, las escalas de tranquilidad $(Z=-2.25, p=.024, r=.15)$ 
y seguridad ( $Z=-2.34, p=.019, r=.15)$ mostraron promedios ligeramente superiores para los hombres, aunque con un tamaño del efecto pequeño. Incluso, la dimensión de emociones negativas no fue la excepción, ya que se presentaron diferencias significativas en tristeza $(Z=-3.49, p=.000$, $r=.23)$, enojo $(Z=-2.57, p=.010, r=.17)$, inseguridad $(Z=-2.85, p=.004$, $r=.18)$ y ansiedad ( $Z=-2.75, p=.006, r=.18)$, donde nuevamente los hombres mostraron puntuaciones más adecuadas.

\section{Discusión}

Los resultados de este estudio muestran diferencias significativas en cuanto a empatía y equilibrio emocional con relación al grado escolar al que pertenecen los participantes, siendo lo opuesto en las emociones restantes, es decir, los estudiantes que han ingresado recientemente a la Licenciatura en Psicología muestran mejores puntajes en las dimensiones de afrontamiento emocional y emociones positivas, lo que supondría que al avanzar en la trayectoria de su formación académica, estos puntajes deberían aumentar. Este resultado sugiere la posibilidad, que la formación académica del psicólogo no está impactando en la habilidad para el desarrollo de inteligencia emocional, competencia esperada en la percepción social además de necesaria para su óptimo ejercicio profesional, tal como lo describe (Barrraza-López, 2015; Echeburúa et al., 2005; Serrano-García, 2015). Lo anterior, invita a discutir sobre la actualización del currículo en la formación del psicólogo, ya que las habilidades como resiliencia, empatía, asertividad y autoestima resultan hoy en día fundamentales para la profesión al momento de relacionarse con los pacientes (Barón \& Benítez, 2020; Cabrera et al., 2010).

Sin embargo, desde otra perspectiva, este resultado también puede sugerir el desarrollo de madurez emocional y de autoprotección ante el enfrentamiento que vive como psicólogo con el sufrimiento humano; así como, de un correcto manejo de empatía humana, para mostrarse objetivo ante su función como profesional de la salud mental (González-Serna et al., 2014; Pérez-González \& García-Ros, 2017).

El contexto de interacción emocional entre los profesionales de la salud mental, y las personas a las que se brinda atención, se retroalimenta de manera recíproca y se ve influido por cogniciones y comportamientos recursivos, teniendo un impacto en todo lo que acontezca en ese espacio de acompañamiento psicoemocional (Ceberio \& Linares, 2005; Fabregó Claparols \& Viñas Millán, 2010). Por ello, autogestionar de forma apropiada, sus emociones como psicólogos, posibilita un desempeño de competencias profesionales adecuadas y flexibles a lo que requiera la persona o usuarios de su profesión (Best \& Pairetti, 2014a).

La empatía visualizada como la capacidad de poder participar en la realidad del otro, ponerse en su situación, captar mensajes verbales y no verbales de los demás, identificar, comprender y respetar cómo otro individuo se siente y ver lo que tienen en común ha resultado en el caso del afrontamiento emocional la que presenta resultados deseables de acuerdo a la literatura señada. No obstante, si se visualizan los resultados 
entre el primer y último año las valoraciones disminuyen al igual que en los hallazgos realizados por Baron y Benítez (2020) y González-Serna et al. (2014) quienes identifican que los niveles de empatía disminuyen progresivamente sobre todo en estudiantes que realizan prácticas de tipo clínico. Más aún, cuando se encuentran mayormente en contacto con pacientes que presentan experiencias de enfermedad y sufrimiento.

De manera agregada hay que señalar que la empatía es fundamental para el caso del psicólogo, quien debe desarrollar dicha habilidad emocional que resulta necesaria para dar un servicio de calidad pudiendo así, reconocer las emociones presentes en los otros y, permite entrar en el mundo del otro, estableciéndose un vínculo y de está manera pueda construir un ambiente seguro dentro de la atención psicológica (Bohart \& Greenberg, 1997 y Norcross \& Wampold, 2011 en Muñoz Aguirre, 2020).

Con relación al equilibrio emocional, Menéndez (2011) vincula el ingreso a la universidad con la felicidad ante el logro académico, situación que posiciona a los de nuevo ingreso con mejores puntajes ante los universitarios que se encuentran en el último año y que están próximos a egresar, que en términos emocionales presentan más sentimientos negativos que positivos en comparación con los de nuevo ingreso. En este sentido, se coincide con Garaigordobil (2013) quien sostiene que conforme se avanza en el desarrollo, la capacidad de evaluación de las emociones aumenta, especialmente si se trata de una transición de la adolescencia a la adultez. Por lo tanto, las valoraciones no necesariamente representan el detrimento de la habilidad emocional, sino un estado de transición hacia la madurez emocional. En este sentido, se puede sugerir que una persona con inteligencia emocional conoce su forma de ser, reconoce sus emociones y las ajusta a la situación, está atenta a lo que sucede a su alrededor, se preocupa por tomar las mejores decisiones para sí misma y para quienes le rodean (Castillo Castañeda, 2016).

Asimismo, los estudiantes de Psicología requieren de habilidades de inteligencia emocional, y aunque acudir a trabajos terapéuticos personales, como parte de su formación académica, son muestra de que se encuentran comprometidos con su formación, es imprescindible brindar mayor atención al tema, pues no se debe olvidar que la función de un plan de estudios sustentado en el modelo de competencias es, llevar la vida real al aula, y que el futuro egresado sepa resolver las problemáticas circundantes, y que mejor realidad que la vida personal (Rábago de Ávila et al., 2019). Lo que deja como tarea pendiente realizar un trabajo de autoanálisis y crítica, con el fin de mejorar la manera en que los conocimientos de la disciplina psicológica se están aproximando al estudiante. Tal como menciona Jiménez González y Báez (2017) se requiere que el profesional de la Psicología reflexione permanentemente los contenidos y procesos de su formación inicial, de su práctica y de las condiciones donde lleva a cabo la implementación. 


\section{Conclusiones}

Finalmente, a partir de los argumentos anteriores podemos concluir que existen diferencias significativas de inteligencia emocional entre los estudiantes de primer y cuarto año de la carrera de psicología, así como también diferencias significativas por sexo, comprobandoce así la hipótesis planteada y rechazando la hipotesis nula.

Sin embargo, los resultados muestran que se requiere mejorar el autoconocimiento y autogestión de las emociones, como habilidades propias de la inteligencia emocional en los estudiantes de Psicología, aunque el entrenamiento profesional implique la disminución de algunas habilidades emocionales en un sentido de autoprotección. Está mejora no se le debe atribuir únicamente a la escuela formadora, pero si se puede sugerir trabaje para desarrollarlas y potenciarlas. Queda claro que, tal como menciona Castillo Castañeda et al. (2016) y Rábago de Ávila et al. (2019), la inteligencia emocional puede llegar a facilitar u obstaculizar el desempeño personal, académico y profesional, y aunque sean identificadas emociones de carácter negativo, estas ayudarán a relacionarse y adaptarse en la vida escolar. En este sentido, Rábago de Ávila et al. (2019, p. 20) con relación a ello, explica que esta habilidad "puede facilitar el tránsito de los futuros profesionistas en su trayecto de formación universitaria, el inadecuado manejo de las mismas puede llegar a repercutir tanto en su desempeño académico, personal y futuro laboral".

Así pues, el psicólogo debe disponer, o bien, desarrollar en este campo habilidades socioemocionales que le permitan un mayor control emocional y le permitan mejorar sus destrezas, la calidad del servicio que brinda y los conocimientos que utiliza (Barón \& Benítez, 2020). Tal como expresan Ruiz et al. (2008, p. 149) "los contextos laborales parecen requerir no sólo profesionales competentes intelectualmente, sino con las suficientes habilidades para manejar las diversas situaciones y retos que plantea la vida laboral".

Respecto a las limitación del estudio, se puede señalar que se han trabajado con muestras de reciente ingreso a la universidad y próximas a egresar, por lo que la inclusión de quienes cursan grados intermedios resulta necesario para identificar la existencia de diferencias en el nivel de desarrollo de inteligencia emocional por grados escolares y visualizar el punto de detrimento de las habilidades que se han mostrado disminuidas en la comparación del inicio y culminación del trayecto escolar del psicólogo en formación. Como aspecto de mejora, se sugiere replicarlo en diferentes universidades que ofrezcan en su oferta profesional la carrera de Psicología para profundizar en el análisis de la profesión y poder visualizar los distintos escenarios educativos. Por otra parte, se recomienda el uso de otras pruebas estadísticas, así como realizar mediciones con otras profesiones dentro de la misma facultad para observar si la situación es específica de la Carrera de Psicología o también corresponde al resto de las opciones profesionales que se desarrollan en la misma facultad. 


\section{Referencias}

Antonio-Agirre, I. A., Izaguirre, L. A., Etxaniz, I. E., \& Maya, M. S. (2015). Capacidad predictiva del autoconcepto y la inteligencia emocional en el ajuste escolar autopercibido. Bordón. Revista de Pedagogía, 67(4), 9-26. h ttp://dx.doi.org/10.13042/Bordon.2015.67401

Baron, A. P., \& Benítez, H. (2020). Exigencias de la profesión del psicólogo en el ámbito emocional en los alumnos de la Carrera de Psicología. ARANDU UTIC, 7(1), 119-143.

Barraza-López, R. (2015). Perspectivas acerca del rol del psicólogo educacional: propuesta orientadora de su actuación en el ámbito escolar. Actualidades Investigativas en Educación, 15(3), 590-610. https://doi.org/10.15517/ai e.v15i3.21070

Bello-Carrasco, I., \& Villavicencio-Navia, K. (2015). La formación del psicólogo como investigador en el Ecuador. Dominio de las Ciencias, 1(1), 108-118.

Belloch Fuster, A. (2005). ¿Qué es un psicólogo clínico?: En torno a la preparación, competencias y desarrollos de una profesión. Análisis y Modificación de Conducta, 21(136-137), 235-272. http://dx.doi.org/10.3 3776/amc.v31i136-137.2188

Best, S., \& Pairetti, C. (2014a). La inteligencia emocional en la formación del psicólogo: su emergencia y construcción como objeto de investigación. Revista Cientifica de UCES, 18(1), 13-36.

Best, S., \& Pairetti, C. (2014b). La inteligencia emocional en la formación del psicólogo: su emergencia y construcción como objeto de investigación. VI Congreso Internacional de Investigación y Práctica Profesional en Psicología XXI Jornadas de Investigación Décimo Encuentro de Investigadores en Psicología del MERCOSUR, Universidad de Buenos Aires, Buenos Aires.

Cabrera, P., Larraín, A., Moretti, R., Manieu, M. A., \& Energici, M. A. (2010). La formación en psicología desde una perspectiva de competencias. Una contribución para el mejoramiento de la formación universitaria en chile. Calidad en la Educación, (33), 183-223. http://dx.doi.org/10.31619/cale du.n33.143

Calero, A. D., Barreyro, J. P., Formoso, J., \& Injoque-Ricle, I. (2018). Inteligencia emocional y necesidad de pertenencia al grupo de pares durante la adolescencia. Subjetividad y Procesos Cognitivos, 22(2), 38-56.

Cardona Carmona, H. (2008). Consideraciones acerca de la educación virtual como comunidad de relaciones afectivo-valorativas. Revista Iberoamericana de Educación, 46(7), 1-10. https://doi.org/10.35362/rie4 671915

Carpio Fernández, M. D. L. V. C. (2019). Perfiles de inteligencia emocional y síntomas externalizantes e internalizantes en la adultez emergente. Electronic Journal of Research in Education Psychology, 17(48), 317-334. h ttp://dx.doi.org/10.25115/ejrep.v17i48.2298

Castillo Castañeda, G. (2016). Competencias emocionales para contribuir a la formación integral de los estudiantes de la UAN (Tesis doctoral). Universidad México-Cubana, México.

Castillo Castañeda, G., Arias Guzmán, A. A., \& Ramírez Vázquez, M. E. (2016). Medición del manejo emocional en estudiantes de nivel superior y su 
importancia en la trayectoria escolar. Revista Educateconciencia, 12(13), 137-150.

Cazalla-Luna, N., \& Molero, D. (2016). Inteligencia emocional percibida, disposición al optimismo-pesimismo, satisfacción vital y personalidad de docentes en su formación inicial. Revista de Investigación Educativa, 34(1), 241-258. https://doi.org/10.6018/rie.34.1.220701

Ceberio, M. R., \& Linares, J. L. (2005). Ser y hacer en terapia sistémica. La construcción del estilo terapéutico. Paidós Ibérica.

Del Rosal Sánchez, I. D., Dávila Acedo, M. A., Sánchez Herrera, S., \& Bermejo García, M. L. B. (2016). La inteligencia emocional en estudiantes universitarios: diferencias entre el grado de maestro en educación primaria y los grados en ciencias. Revista INFAD de Psicología. International Journal of Developmental and Educational Psychology, 2(1), 51-62. https://doi.or g/10.17060/ijodaep.2016.n1.v2.176

Delors, J., Amagi, I., Carneiro, R., Chung, F., Geremek, B., Gorham, W., ... \& Nanzhao, Z. (1997). La educación encierra un tesoro: informe para la UNESCO de la Comisión Internacional sobre la Educación para el Siglo Veintiuno. Santillana Ediciones UNESCO.

Echeburúa, E., de Corral, P., \& Salaberría, K. (2005). Reflexiones ante la formación del psicólogo. Retos de futuro. Análisis y Modificación de Conducta, 31(136-137), 175-188. http://dx.doi.org/10.33776/amc.v31i 136-137.2185

Echeburúa, E., Salaberría, K., Corral, P. D., \& Cruz-Sáez, S. (2012). Funciones y ámbitos de actuación del psicólogo clínico y del psicólogo general sanitario: una primera reflexión. Behavioral Psychology/Psicología Conductual, 20(2), 423-435.

Fabregó Claparols, E., \& Viñas Millán, A. V. (2010). Las emociones del terapeuta sistémico en juego. Redes: Revista de Psicoterapia Relacional e Intervenciones Sociales, 23, 129-148.

Fernández-Berrocal, P., \& Pacheco, N. E. (2002). La inteligencia emocional como una habilidad esencial en la escuela. Revista Iberoamericana de Educación, 29(1), 1-6. https://doi.org/10.35362/rie2912869

Fragoso-Luzuriaga, R. (2015). Inteligencia emocional y competencias emocionales en educación superior, ¿un mismo concepto? Revista Iberoamericana de Educación Superior, 6(16), 110-125.

Garaigordobil, M. (2013). Sexismo y alexitimia: correlaciones y diferencias en función del género, la edad, y el nivel de estudios. Anales de Psicologial Annals of Psychology, 29(2), 368-377. https://doi.org/10.6018/analesps.2 9.2.132261

Gartzia, L., Aritzeta Galan, A., Balluerka Lasa, N., \& Barbera Heredia, E. (2012). Inteligencia emocional y género: más allá de las diferencias sexuales. Anales de Psicología, 28(2), 567-575. https://doi.org/10.6018/analesps.28.2.124 111

González-Serna, J. M. G., Serrano, R. R., Martín, M. S. M., \& Fernández, J. M. A. (2014). Descenso de empatía en estudiantes de enfermería y análisis de posibles factores implicados. Psicología Educativa, 20(1), 53-60. https://d oi.org/10.1016/j.pse.2014.05.007

Gutiérrez Carmona, M. G., \& Expósito López, J. E. (2015). Autoconcepto, dificultades interpersonales, habilidades sociales y conductas asertivas en 
adolescentes. Revista Española de Orientación y Psicopedagogía, 26(2), 42-58. https://doi.org/10.5944/reop.vol.26.num.2.2015.15215

Gutiérrez Rodríguez, J., Abril Herrero, M. F., Zorzo, M. E., García Torres, M. Á., González Arranz, E., \& Sahagún Flores, L. M. (2018). La actuación profesional de la psicología en las residencias de personas mayores. Revista INFAD de Psicología. International Journal of Developmental and Educational Psychology, 4(1), 107-120. https://doi.org/10.17060/ijodaep .2018.n1.v4.1277

Jiménez González, J. E., \& Báez, B. F. (2017). Formación y regulación profesional de la Psicología en Educación: un desafío a nuestro alcance. Informació Psicologica, (113), 33-47. http://dx.medra.org/10.14635/IPSI C.2017.113.2

Menéndez, I. (2011). El equilibrio emocional. Espasa.

Muñoz Aguirre, C. B. M. (2020). Función de la empatía en el trabajo de psicólogos y psicoterapeutas. Revista Scientific, 5(Ed. Esp.), 146-164. http s://doi.org/10.29394/Scientific.issn.2542-2987.2020.5.E.7.146-164

Paez Cala, M. L., \& Castaño Castrillón, J. J. (2015). Inteligencia emocional y rendimiento académico en estudiantes universitarios. Psicología desde el Caribe, 32(2), 268-285. http://dx.doi.org/10.14482/psdc.32.2.5798

Pérez-Bonet, G., \& Velado-Guillén, L. A. V. (2017). Inteligencia emocional percibida (IEP) en el alumnado universitario de educación: análisis comparativo por género y grado. EA, Escuela Abierta: Revista de Investigación Educativa, (20), 23-34. https://doi.org/10.29257/EA20.20 17.03

Pérez-González, F., \& García-Ros, R. (2017). Los profesionales de la psicología de la educación en el siglo XXI: un escenario complejo. Informació Psicologica, (113), 48-60. http://dx.medra.org/10.14635/IPSIC.2017.11 3.3

Rábago de Ávila, M. R., Castillo Castañeda, G. C., \& Pérez Sánchez, L. P. (2019). Manejo emocional en estudiantes de psicología. Educación y Ciencia, 8(51), 19-29.

Rodrigo López, M. J. R. (2017). La formación de profesionales en ámbitos educativos no formales: una apuesta por el ámbito de la intervención y mediación familiar. Informació Psicologica, (113), 85-93. http://dx.medra .org/10.14635/IPSIC.2017.113.5

Ruiz, M., Jaraba, B., \& Romero Santiago, L. (2008). La formación en psicología y las nuevas exigencias del mundo laboral: competencias laborales exigidas a los psicólogos. Psicología desde el Caribe, (21), 136-157.

Salovey, P., \& Mayer, J. D. (1990). Emotional intelligence. Imagination, Cognition and Personality, 9(3), 185-211. https://doi.org/10.2190/DUG G-P24E-52WK-6CDG

Santoya Montes, Y., Garcés Prettel, M., \& Tezón Boutureira, M. (2018). Las emociones en la vida universitaria: análisis de la relación entre autoconocimiento emocional y autorregulación emocional en adolescentes y jóvenes universitarios. Psicogente, 21(40), 422-439. https:/ /doi.org/10.17081/psico.21.40.3081

Secretaría de Educación Pública. (2017). Aprendizajes clave para la educación integral. México.

Serrano-García, I. (2010). Retos en la formación del psicólogo/a comunitario/a en las Américas. Ciencias Psicológicas, 4(2), 225-234. 
Suárez Colorado, Y. S., \& Wilches Bisval, C. W. (2015). Habilidades emocionales en una muestra de estudiantes universitarios: las diferencias de género. Educación y Humanismo, 17(28), 119-132. https://doi.org/10 .17081/eduhum.17.28.1170

Suárez Colorado, Y. S., Guzmán García, K. G., Medina Alfonso, L. M., \& Ceballos Ospino, G. C. (2012). Características de inteligencia emocional y género en estudiantes de psicología y administración de empresas de una universidad pública de Santa Marta, Colombia: un estudio piloto. Duazary, 9(2), 132-139.

Valadez Sierra, M. D. L. D. V., Borges del Rosal, M. Á., Ruvalcaba Romero, N., Villegas, K., \& Lorenzo, M. (2013). La inteligencia emocional y su relación con el género, el rendimiento académico y la capacidad intelectual del alumnado universitario. Electronic Journal of Research in Educational Psychology, 11(2), 395-412. http://dx.doi.org/10.14204/ejrep.30.12204

Vivas García, M. (2003). La educación emocional: conceptos fundamentales. Sapiens. Revista Universitaria de Investigación, 4(2), 0. 